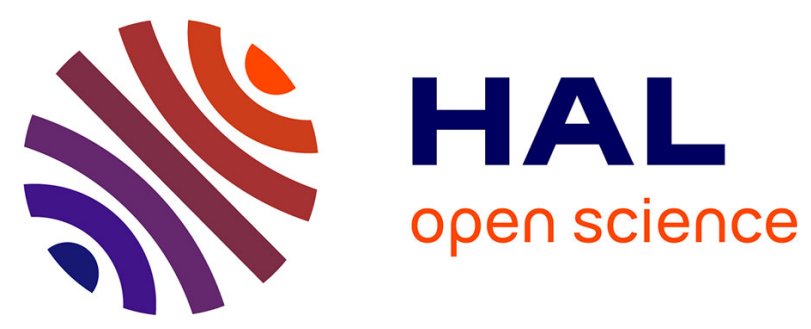

\title{
Looking for the agent: an investigation into consciousness of action and self-consciousness in schizophrenic patients
}

Elena Daprati, Nicolas Franck, Nicolas Georgieff, Joëlle Proust, Elisabeth Pacherie, Jean Dalery, Marc Jeannerod

\section{To cite this version:}

Elena Daprati, Nicolas Franck, Nicolas Georgieff, Joëlle Proust, Elisabeth Pacherie, et al.. Looking for the agent: an investigation into consciousness of action and self-consciousness in schizophrenic patients. Cognition, 1997, 65, pp.71-86. ijn_00000257v2

\section{HAL Id: ijn_00000257 \\ https://hal.science/ijn_00000257v2}

Submitted on 12 Mar 2005

HAL is a multi-disciplinary open access archive for the deposit and dissemination of scientific research documents, whether they are published or not. The documents may come from teaching and research institutions in France or abroad, or from public or private research centers.
L'archive ouverte pluridisciplinaire HAL, est destinée au dépôt et à la diffusion de documents scientifiques de niveau recherche, publiés ou non, émanant des établissements d'enseignement et de recherche français ou étrangers, des laboratoires publics ou privés. 


\title{
Looking for the agent: an investigation into consciousness of action and self-consciousness in schizophrenic patients
}

\author{
E. Daprati ${ }^{\mathrm{a}, \mathrm{e}}$, N. Franck ${ }^{\mathrm{a}, *}$, N. Georgieff ${ }^{\mathrm{a}, \mathrm{b}}$, J. Proust $^{\mathrm{c}}$, \\ E. Pacherie ${ }^{c}$, J. Dalery ${ }^{\mathrm{d}}$, M. Jeannerod ${ }^{\mathrm{a}}$ \\ anstitut des Sciences Cognitives, CNRS UPR 9075, 8 Avenue Rockefeller, 69373 Lyon, France \\ ${ }^{\mathrm{b}}$ Jeune Equipe 1882, Université Lyon-1, Lyon, France \\ ${ }^{\mathrm{c}}$ CREA, Paris, France \\ ${ }^{\mathrm{d}}$ Hopital du Vinatier, Lyon, France \\ ${ }^{\mathrm{e}}$ Institute of Human Physiology, University of Parma, Via Gramsci 14, 43100 Parma, Italy
}

Received 21 July 1997; accepted 8 October 1997

\begin{abstract}
The abilities to attribute an action to its proper agent and to understand its meaning when it is produced by someone else are basic aspects of human social communication. Several psychiatric syndromes, such as schizophrenia, seem to lead to a dysfunction of the awareness of one's own action as well as of recognition of actions performed by others. Such syndromes offer a framework for studying the determinants of agency, the ability to correctly attribute actions to their veridical source. Thirty normal subjects and 30 schizophrenic patients with and without hallucinations and/or delusional experiences were required to execute simple finger and wrist movements, without direct visual control of their hand. The image of either their own hand or an alien hand executing the same or a different movement was presented on a TV-screen in real time. The task for the subjects was to discriminate whether the hand presented on the screen was their own or not. Hallucinating and deluded schizophrenic patients were more impaired in discriminating their own hand from the alien one than the non-hallucinating ones, and tended to misattribute the alien hand to themselves. Results are discussed according to a model of action control. A tentative description of the mechanisms leading to action consciousness is proposed. (C) 1997 Elsevier Science B.V.
\end{abstract}

Keywords: Schizophrenia; Hallucinations; Consciousness; Representation of action; Motor representations

\footnotetext{
* Corresponding author. Fax: +33 478 777286; e-mail: franck@ rockefeller1.univ-lyon1.fr
} 


\section{Introduction}

A fundamental problem is raised by the mechanism of how one becomes aware of one's own actions, and how one's actions are distinguished from those of other people. This mechanism is critical for a number of reasons. First, the ability to make a distinction between the acting self and the acting other is one way by which the self feels distinct from other individuals and self-consciousness is built. Second, the ability to recognise the meaning of an action when it is produced by someone else and to attribute it to its proper agent is a basic function underlying human social communication.

Research on these points has been undertaken within several different frameworks. The study of mental and cognitive development is one of those. Researchers in this field have set the stage for a renewal of developmental studies, by demonstrating the appearance, in early childhood, of a special ability of the individual to attribute to others mental states differing from his/her own, and to infer the content of these mental states from his/her own mental content (the so-called 'theory of mind', Leslie, 1987). The ability to imitate and to learn by observation is another critical developmental function, which might relate to the same basic mechanism (Meltzoff, 1995). This highly influential research has recently merged with other work in normal adult subjects, aimed at determining the neural mechanisms of mental representations, particularly in the context of action generation. The notions of internal models and internal simulation borrowed from computational neuroscience (see Wolpert et al., 1995) have now become a conceptual tool for studying the early stages of action generation, such as intending, for example. Experiments using brain mapping techniques have shown that merely observing an action performed by someone else is no less a powerful way of eliciting brain activity than other cognitive motor states, such as mentally simulating or preparing to execute that same action. These results (see Section 4) raise the point of the differences and similarities of motor representations activated during these states.

The study of mental disorders is another possible framework for understanding the problems of consciousness of action and self-consciousness. Some of the symptoms observed in schizophrenia, specifically the positive symptoms typical of the acute stage of the disease, seem to be relevant to a dysfunction of the awareness of one's own action and to a dysfunction of recognition of actions performed by others. One possible, general explanation for this difficulty would arise from a disability in attributing meaning to external events, itself due to a weakening of inhibitory processes crucial for consciously focusing attention (see Gray et al., 1991). Another, more specific, hypothesis is that of Frith, implying that schizophrenics fail in monitoring their willed intentions, including those related to the expression of thought (Frith, 1987, 1992). They fail to attribute elements arising from their long-term memory, from which they form their goals and plans, to their real origin. The consequence of this failure in monitoring makes them unable to disentangle 'intentions' arising from external stimuli, from those generated as a consequence of their own cognitive functioning. Positive symptoms such as inser- 
tion of thought, hallucinations and delusion of control would directly derive from this difficulty.

Verbal hallucinations seem to be liable to this explanation. According to Frith (1996), the normal mechanism for attributing thought to its internal origin would be a comparison between the executive commands leading to speech and the anticipated sensory consequences of these commands. Normally, execution of the speech motor commands implies that the related sensory signals will be inhibited (Creutzfeldt et al., 1989). During verbal hallucinations in schizophrenic patients, by contrast, the sensory areas for language remain active, which suggests that the cancellation process does not operate. The nervous system in these patients behaves as if it were actually processing the speech of an external speaker. Hence their perception of their own thinking as originating from the outside world (Silbersweig et al., 1995; McGuire et al., 1996).

In the above situation, the patients attributed their own action to an external source. This tendency, however, only corresponds to part of the positive symptomatology in schizophrenia. The same patients may experience the reverse as well, i.e. they may experience the action of others as a consequence of their own intentions.

In the present study we compared groups of normal controls and of patients with and without positive symptoms, when required to make agency judgements about a hand movement originating either from themselves or from an experimenter. Our results showed that patients with hallucinations or delusional control, when faced with hand movements of uncertain origin (e.g. an alien hand performing the same movement as them), systematically considered the movements as theirs. This striking result raises the point of which cues are normally used for producing conscious judgements about the origin of an action.

\section{Method}

The purpose of the study was approved by the local ethical committee (CCPPRBLyon). All subjects gave informed consent for participation in the experiment.

\subsection{Participants}

Sixty subjects participated, 30 schizophrenic patients and 30 normal control subjects.

\subsubsection{Schizophrenic patients}

The patients (24 males and 6 females, mean age 34.5) were diagnosed as schizophrenics according to the DSM-IV. Eighteen received diagnosis of paranoid schizophrenia, seven of undifferentiated schizophrenia, and five of residual schizophrenia. According to clinical evaluation (Andreasen's scales for assessment of positive and negative symptoms; Andreasen, 1983, 1984), a clear pattern of hallucinations was present in 13 patients. Delusion of control was reported 
by seven patients. In six patients delusion of control was associated with presence of hallucinations. All patients were under medication during the period of the study, and medication averaged 427.2 (SD 320.4) chlorpromazine equivalents. Estimated mean illness duration was 12.3 years. A summary of patients' data is given in Table 1.

All but four patients were right-handed according to the Edinburgh Inventory (Oldfield, 1971). Vision was normal or corrected to normal. Patients' mean IQ, as estimated by the score at the Raven Progressive Matrices PM47, was 91.7. Shortterm memory span was 6.4 and 3.5, forward and backwards, respectively (Wechsler, 1981). Language comprehension, as assessed by the Token Test (De Renzi and Vignolo, 1962) was good (mean score 33.2/36).

Table 1

Patient details

\begin{tabular}{|c|c|c|c|c|c|c|c|c|}
\hline Name & Sex & Age & Handedness & $\begin{array}{l}\text { Classification } \\
\text { DSM-IV }\end{array}$ & $\begin{array}{l}\text { Estimated } \\
\text { illness } \\
\text { duration } \\
\text { (in years) }\end{array}$ & $\begin{array}{l}\text { Medication } \\
\text { (Chlorpro- } \\
\text { mazine } \\
\text { equivalents) }\end{array}$ & $\begin{array}{l}\text { Hallu- } \\
\text { cinations }\end{array}$ & $\begin{array}{l}\text { Delusion } \\
\text { of } \\
\text { Control }\end{array}$ \\
\hline VAC & M & 23 & Mixed & Paranoid & 6 & 700 & +++ & - \\
\hline $\mathrm{BOU}$ & M & 29 & Right-hander & Paranoid & 2 & 260 & +++ & - \\
\hline VEU & M & 22 & Mixed & Residual & 1 & 170 & - & - \\
\hline DIF & M & 21 & Right-hander & Residual & 3 & 50 & - & - \\
\hline PEG & M & 21 & Right-hander & Residual & 2 & 360 & - & - \\
\hline SEQ & M & 20 & Right-hander & Paranoid & 4 & 1500 & - & + \\
\hline MAT & M & 39 & Left-hander & Paranoid & 17 & 600 & ++ & - \\
\hline LAI & M & 34 & Right-hander & Paranoid & 12 & 250 & ++ & - \\
\hline CAT & M & 41 & Right-hander & Paranoid & 14 & 1080 & ++ & + \\
\hline MAR & $\mathrm{F}$ & 26 & Right-hander & Paranoid & 9 & 533 & + & - \\
\hline KIZ & $\mathrm{F}$ & 27 & Right-hander & Undifferentiated & 5 & 150 & - & - \\
\hline BUA & $\mathrm{F}$ & 58 & Right-hander & Undifferentiated & 23 & 170 & - & - \\
\hline OUR & M & 24 & Right-hander & Paranoid & 5 & 800 & - & - \\
\hline ROU & M & 52 & Right-hander & Paranoid & 32 & 150 & - & - \\
\hline CHA & M & 37 & Left-hander & Residual & 21 & 400 & - & - \\
\hline THE & M & 57 & Right-hander & Undifferentiated & 32 & 700 & ++ & - \\
\hline BEN & M & 44 & Right-hander & Undifferentiated & 17 & 90 & - & - \\
\hline BAR & M & 42 & Right-hander & Paranoid & 26 & 500 & + & + \\
\hline TRA & M & 25 & Right-hander & Undifferentiated & 5 & 400 & - & - \\
\hline FER & M & 35 & Right-hander & Paranoid & 13 & 400 & +++ & + \\
\hline NOU & M & 21 & Right-hander & Residual & 4 & 300 & - & - \\
\hline CAM & $\mathrm{F}$ & 43 & Right-hander & Undifferentiated & 18 & 150 & - & - \\
\hline OLL & $\mathrm{F}$ & 20 & Right-hander & Paranoid & 5 & 600 & ++ & + \\
\hline TIB & M & 24 & Right-hander & Paranoid & 1 & 250 & - & - \\
\hline BOJ & M & 48 & Right-hander & Paranoid & 15 & 420 & +++ & + \\
\hline AUA & $\mathrm{F}$ & 33 & Right-hander & Paranoid & 4 & 300 & - & - \\
\hline MES & M & 32 & Right-hander & Paranoid & 9 & 250 & + & + \\
\hline BEI & M & 60 & Right-hander & Paranoid & 27 & 130 & - & - \\
\hline MOU & M & 41 & Right-hander & Paranoid & 21 & 825 & ++ & - \\
\hline POL & M & 36 & Right-hander & Undifferentiated & 17 & 330 & - & - \\
\hline
\end{tabular}


From the experimental group, 17 patients (six with hallucinations, five with hallucinations and delusion of control, and six without hallucinations nor delusion of control) could be recruited for a further session in which the integrity of visual perception was assessed by means of a subset of the Birmingham Object Recognition Battery (BORB; Riddoch and Humphreys, 1993). Four tests investigated the ability of the patients to process basic properties of visual stimuli, such as length (line length match test A), size (circle size match test A), orientation (line orientation match test A) and location (position of gap match test A). In each test a samedifferent judgement on a pair of stimuli is required. Two tests assessed the capacity of the patients to derive viewpoint-invariant visual information, and to access stored knowledge about specific items from vision. In the first test, the patient had to choose which of two pictures represents the same object of a target picture despite being depicted from a different viewpoint (Minimal Feature View Task), whereas in the second test the patient had to find which picture belongs to the same category of the target (Item Match Task). The patients performance on the six tests was well within the normal range (line match test $\mathrm{A}=26,7 / 30$, cut-off point 22; circle match test $A=27,4 / 30$, cut-off point 19 ; line orientation match test $A=26,6 / 30$, cut-off point 18; position of gap match test $\mathrm{A}=34 / 40$, cut-off point 24; Minimal Feature View Task $=24,8 / 25$, cut-off point 18,5; Item Match Task $=31,9 / 32$, cut-off point 24).

\subsubsection{Control subjects}

Thirty normal subjects (19 males and 11 females, mean age 28.8 years) volunteered for this study. None of them reported evidence of neurological diseases nor psychiatric problems, as assessed by the Mini International Neuropsychiatric Interview (Lecrubier et al., 1997). All but four subjects were right-handed according to the Edinburgh Inventory (Oldfield, 1971). Vision was normal or corrected to normal. Their mean IQ, as estimated by the score at the Raven Progressive Matrices PM47, was 97.4. Short-term memory span was 6.6 and 4.6, forward and backwards, respectively (Wechsler, 1981). Language comprehension, as assessed by the Token Test (De Renzi and Vignolo, 1962) was normal (mean score 35.4/36).

\subsubsection{Comparison between patients and controls}

A $t$-test for independent samples was carried out in order to verify that control subjects and schizophrenic patients were sufficiently matched. No difference was found between the two groups regarding age and IQ. However, patients performed more poorly than controls at the Token Test $(t=6.909, P<0.0001)$, and showed a reduced backward short-term memory span $(t=+3.047, P<0.004)$.

\subsubsection{Comparisons within the schizophrenics group}

The 13 patients reporting hallucinations did not differ from the rest of the group regarding age, illness duration, therapy and neuropsychological variables. The seven patients with experience of delusion of control were significantly more medicated than the other patients $(t=-2.593, P<0.01)$, their IQ was lower $(t=2.264$, $P<0.03)$ and performance at the Token Test poorer $(t=2.330, P<0.02)$. Finally, 
patients with experience of delusion of control showed a reduced forward short-term memory span $(t=2.500, P<0.02)$ with respect to the other ones.

\subsection{Apparatus and procedure}

Both schizophrenic patients and control subjects were naive as to the purpose of the experiment. An apparatus inspired from Nielsen (1963) was built, a schematic representation of which is shown in Fig. 1. Two cabins were located in a dimly-lit room. Subjects entered the apparatus by one side of the largest cabin $(200 \times 200 \times 70 \mathrm{~cm})$. The technical devices were hidden to them. Subjects sat on a comfortable chair in front of a table (height $80 \mathrm{~cm})$. A rectangular mirror $(30 \times 30$ $\mathrm{cm}$ ) was placed at $40 \mathrm{~cm}$ from the plane of the table, and at $35 \mathrm{~cm}$ from the subjects' frontal plane. This mirror (M1 on Fig. 1) was oriented with an inclination of $30^{\circ}$ on the vertical plane. Subjects positioned their right hand below the mirror. The hand rested either on a support located at approximately $40 \mathrm{~cm}$ from the subjects' frontal plane, or grasped a joystick located at the same position. A camera (distance $150 \mathrm{~cm}$ from the table) connected to a closed-circuit television system filmed the subject's hand through another mirror (M2). Its image appeared on a TV-screen located on a shelf, $15 \mathrm{~cm}$ over the subject's head, and was reflected by mirror M1. Thus, looking

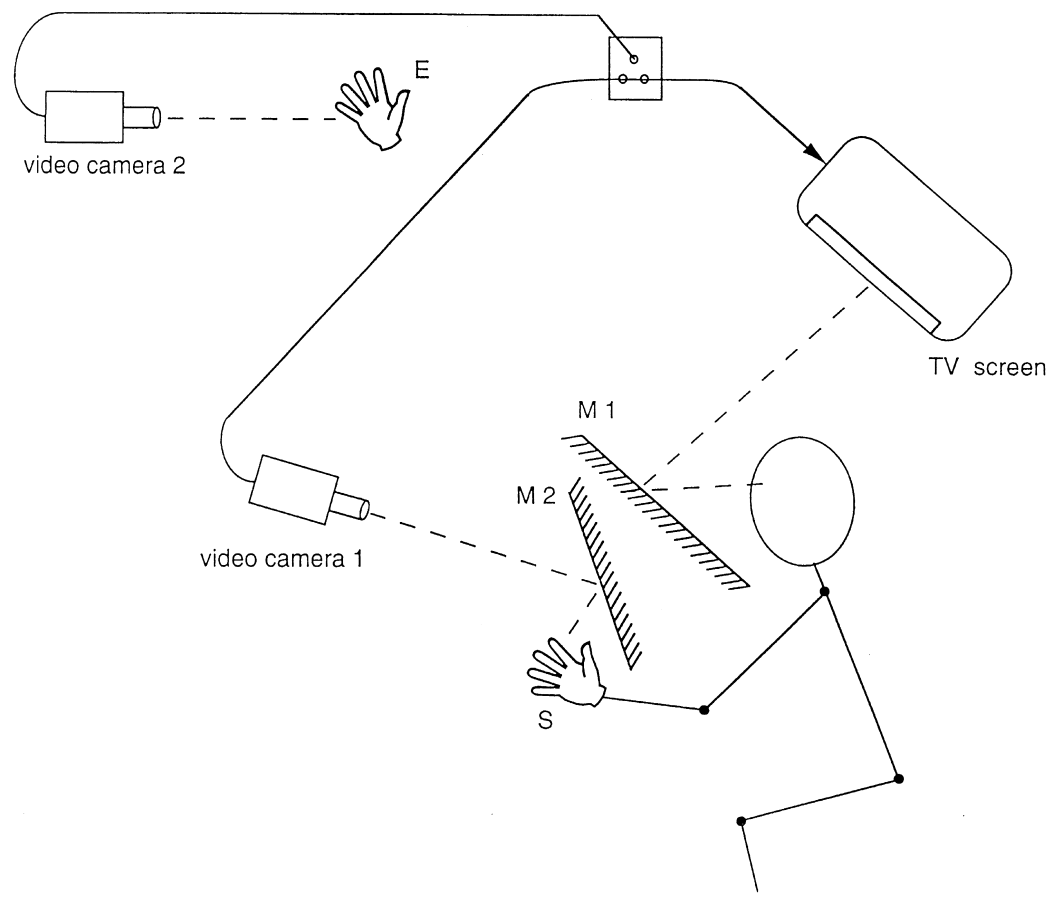

Fig. 1. Schematic representation of the experimental apparatus. S, subject; E, experimenter; M1, mirror one; M2, mirror two. Video cameras 1 and 2 were located in separate cabins. They were not visible to the subjects. Further details in text. 
at the mirror, subjects got the impression that they watched their own hand as through a window.

Close to the subject's cabin, a second cabin $(170 \times 170 \times 70 \mathrm{~cm})$ was devised for the experimenter, whose hand was filmed by a second camera. The cabin was identical to the subjects' one, apart from the fact that no mirror was present. Two control screens were present in the cabin, one showing the image of the subject's hand, the other, of the experimenter's hand. This display allowed the experimenter to exactly match the image of her hand with that of the subject's hand before the beginning of each trial. A special switch allowed the TV-screen to present either the image of the subject's hand or that of the experimenter. The experimenter's and the subject's hands were covered with identical gloves, in order to minimise the effects of gross morphological differences.

The task for the subjects was to perform a requested movement with their right hand, and to monitor its execution by looking at the image on the mirror. The head was free to move during the experiment, and no constraints were imposed on eye-movements. Before the experiment, participants were allowed to freely move the hand below the mirror, until they were familiar with the apparatus. Next, six practice trials were run before the experimental session itself started.

The procedure was as follows: at the beginning of each trial the screen was dark and no image was presented. Then, an advance instruction was given as to which movement to execute, and the image of a hand appeared on the screen. At the same time, a beep produced by a PC was the signal for the subject and the experimenter to execute the requested movement. Once the movement was performed, the screen returned to darkness, within about $1 \mathrm{~s}$, and a question was asked to the subject. The question said: 'You have just seen the image of a moving hand. Was it your own hand? Answer YES if you saw your own hand performing the movement you have been executing. Answer NO in any other case, that is if you doubt that it was your own hand or your own movement.'

Two sets of movements were tested: finger set and wrist set. In the finger set the hand was resting on the support, fist closed. One of four possible movements of the fingers was required in each trial: 1. extend thumb, 2. extend index finger, 3. extend index and middle finger, 4. open hand wide. In the wrist set the hand grasped a joystick, holding it in the vertical position. One of four possible wrist rotations was required in each trial: 1 . forward, 2. backward, 3. left, 4. right.

In each set of movement, one of three possible images of the hand could be presented to the subjects in each trial: 1. their own hand (condition: Subject), 2. the experimenter's hand performing the same movement (condition: Experimenter Same), 3. the experimenter's hand performing a different movement (condition: Experimenter Different).

Twelve trials were run for each hand condition, that is 3 trials for each movement type. All together, 36 trials were run for each set of movements. Order of set presentation was counterbalanced across subjects, whereas movement type and hand presented were randomised in each set. 


\subsection{Data analysis}

Verbal responses were recorded for analysis. Each time subjects and patients did not recognise their own hand, or mis-attributed the alien hand to themselves, an error was recorded. The number of erroneous responses was submitted to analysis. Due to the peculiar nature of the tested variable and to the small number of trials, nonparametric analysis was used to test the interactions between subjects in the different experimental conditions. The Kruskal-Wallis analysis of ranks was used for between groups comparisons, whereas the Friedman's two-way analysis of variance, and the Wilcoxon's matched pairs test were used to test differences between conditions. Pairwise comparisons were assessed by means of the Mann-Whitney $U$-test for comparisons of groups and Wilcoxon's matched pairs test for comparisons of conditions.

\section{Results}

Both control subjects and patients well understood the task requests. Errors in the procedure were not frequent (controls $0.37 \%$; patients $1.02 \%$ ), and included execution of a movement different from the required one, movement start before the gosignal, additional movements or submovements during, or immediately after the required one. The trial was repeated each time one of these errors occurred.

\subsection{Presencelabsence of hallucinations}

Descriptive analyses showed that overall hallucinating patients made more recognition errors (median value: 24) than non-hallucinating patients (11), and controls (5). The Kruskal-Wallis test on total error number revealed that the difference between groups was significant $(P=0.00001)$. Subsequent pairwise comparisons performed using the Mann-Whitney $U$-test showed that both groups of patients produced significantly more errors than the control group (control vs. non-hallucinating $P=0.0135$; control vs. hallucinating $P=0.000009)$. The two groups of patients differed from each other $(P=0.031)$.

At a descriptive level, no difference was evident in distribution of errors in either the finger or the wrist set for each group of subjects (median value - controls: finger set 3 , wrist set 3 ; non-hallucinating patients: finger set 6 , wrist set 6 ; hallucinating patients: finger set 11, wrist set 12). A Wilcoxon's matched pairs test confirmed that errors occurred in the same proportion in the two sets of movements in all subject populations (comparison finger set vs. wrist set, controls $P=0.338$; non-hallucinating $P=0.816$; hallucinating $P=0.102$ ). Therefore, the number of errors recorded in the two sets of movements were pooled for subsequent analysis.

Errors occurred especially in the condition Experimenter Same, in all groups (controls $P=0.000001$; non-hallucinating $P=0.000001$; hallucinating $P=$ 0.00002 in the Friedman test). In the trials when the hand on the screen performed the same movement that was required from the subject but was not his/her own, the 
Table 2

Mean values (standard deviations) for error rate in control subjects and schizophrenic patients. Patients have been divided in two groups according to symptoms

\begin{tabular}{|c|c|c|c|c|c|c|}
\hline & \multicolumn{3}{|l|}{ Finger } & \multicolumn{3}{|l|}{ Wrist } \\
\hline & Subject & Exp. same & Exp. diff. & Subject & Exp. same & Exp. Diff. \\
\hline \multicolumn{7}{|l|}{ (a) Hallucinations } \\
\hline Controls & $0.07(0.36)$ & $3.83(3.23)$ & $0.00(0.00)$ & $0.10(0.30)$ & $3.50(3.49)$ & $0.00(0.00)$ \\
\hline Non-hallucinating & $0.65(1.27)$ & $6.53(4.27)$ & $0.12(0.48)$ & $0.29(0.77)$ & $5.65(4.73)$ & $1.23(2.54)$ \\
\hline Hallucinating & $0.69(1.70)$ & $9.23(3.96)$ & $0.23(0.83)$ & $0.61(1.12)$ & $9.31(2.98)$ & $1.61(2.81)$ \\
\hline \multicolumn{7}{|c|}{ (b) Delusion of control } \\
\hline Controls & $0.07(0.36)$ & $3.83(3.23)$ & $0.00(0.00)$ & $0.10(0.30)$ & $3.50(3.49)$ & $0.00(0.00)$ \\
\hline Non-delusional & $0.74(1.60)$ & $7.30(4.26)$ & $0.22(0.74)$ & $0.43(1.04)$ & $6.30(4.46)$ & $1.74(2.89)$ \\
\hline Delusional & $0.43(0.79)$ & $9.00(4.47)$ & $0.00(0.00)$ & $0.43(0.53)$ & $10.29(2.63)$ & $0.29(0.76)$ \\
\hline
\end{tabular}

median for error rate was 5 in the control group, 11 in the non-hallucinating group and 22 in the hallucinating group, whereas virtually no judgement errors occurred when subjects saw their own hand, or a hand performing a different movement. Differences between the three groups for the condition Experimenter Same showed a strong trend to significance (comparison controls vs. non-hallucinating $P=0.06$; controls vs. hallucinating $P=0.00006$; non-hallucinating vs. hallucinating $P=0.05$ in the Mann-Whitney $U$ test). Results are summarised in Table 2a and Fig. 2a.

\subsection{Presencelabsence of delusion of control}

Descriptive analyses showed that overall patients experiencing delusion of control made more recognition errors (median value: 24) than the rest of the patients (18), and controls (five). The Kruskal-Wallis test on total error number revealed that the difference between groups was significant $(P=0.0001)$. Subsequent pairwise comparisons performed using the Mann-Whitney $U$-test showed that both groups of patients produced significantly more errors than the control group (control vs. nondelusional $P=0.0006$; control vs. delusional $P=0.0006)$. The two groups of patients did not differ from each other $(P=0.3)$.

At a descriptive level, no difference was evident in distribution of errors in either the finger or the wrist set for each group of subjects (median value - controls: finger set 3, wrist set 3; non-delusional patients: finger set 9, wrist set 8; delusional patients: finger set 11, wrist set 12). A Wilcoxon's matched pairs test confirmed that errors occurred in the same proportion in the two sets of movements in all subject populations (comparison finger set vs. wrist set, controls $P=0.338$; nondelusional $P=0.903$; delusional $P=0.338$ ). Therefore, the number of errors recorded in the two sets of movements were pooled for subsequent analysis.

Errors occurred especially in the condition Experimenter Same, in all groups (controls $P=0.000001$; non-delusional $P=0.000001$; delusional $P=0.002$ in the Friedman test). In the trials when the hand on the screen performed the same movement that was required from the subject but was not his/her own, the median for 

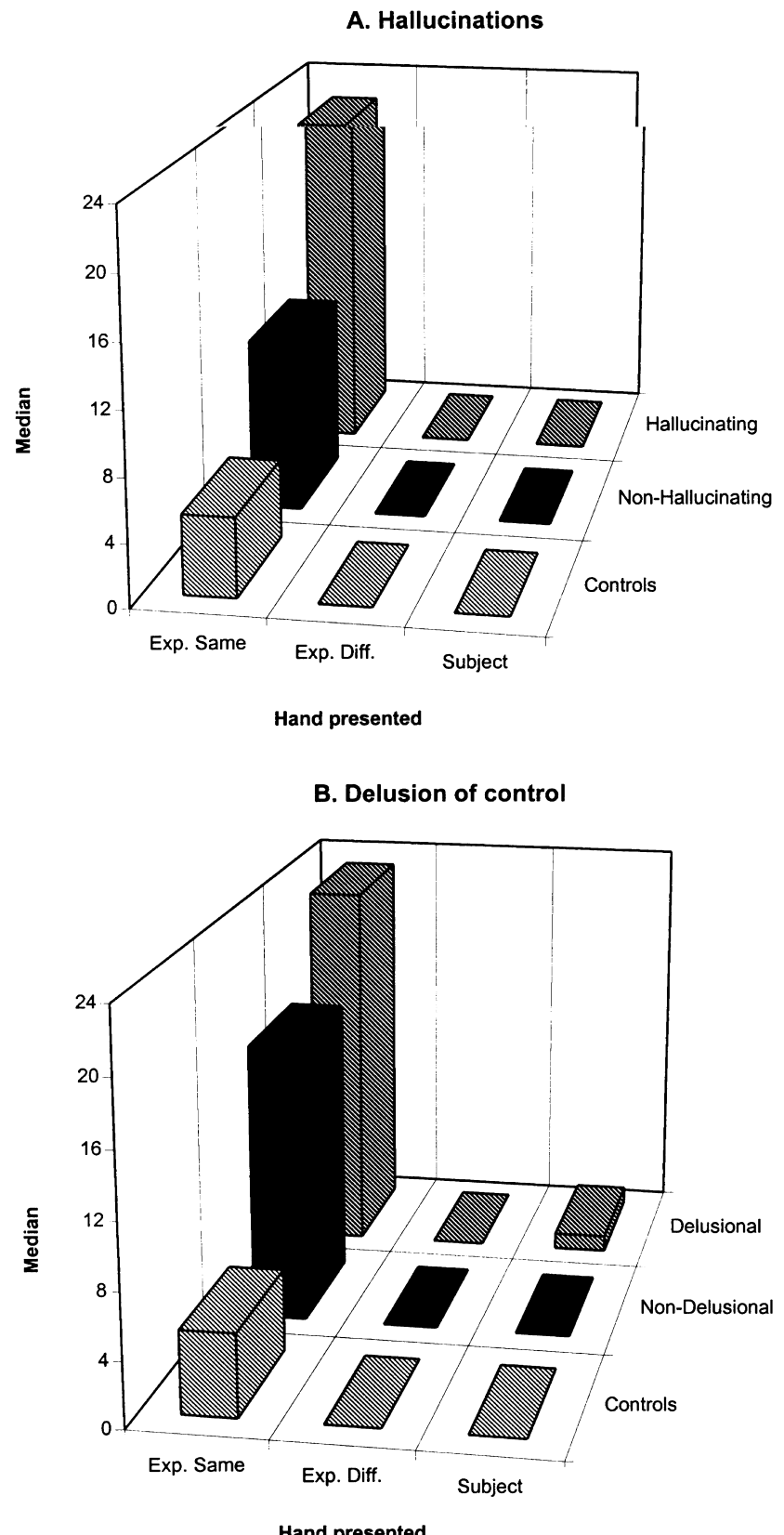

Fig. 2. Number of errors reported for control subjects and schizophrenic patients (median values). a. Schizophrenic patients have been divided in two groups according to the presence $(n=13)$ or absence $(n=17)$ of hallucinations. b. Schizophrenic patients have been divided in two groups according to the presence $(n=7)$ or absence $(n=23)$ of delusion of control. 
error rate was 5 in the control group, 17 in the non-delusional group and 23 in the delusional group, whereas virtually no judgement errors occurred when subjects saw their own hand, or a hand performing a different movement. Differences between the three groups for the condition Experimenter Same showed a strong trend to significance (comparison controls vs. non-delusional $P=0.006$; controls vs. delusional $P=0.001$; non-delusional vs. delusional $P=0.07$ in the Mann-Whitney $U$ test). Results are summarised in Table $2 b$ and Fig. $2 b$.

\section{Discussion}

In the present experiment, normal control subjects were able to unambiguously determine whether the moving hand seen on the screen was theirs or not, in two conditions. First, when they saw their own hand (trials from the condition Subject), they correctly attributed the movement to themselves. Second, when they saw the experimenter's hand performing a movement which departed from the instruction they had received (condition Experimenter Different), they denied seeing their own hand. By contrast, their performance degraded in the condition Experimenter Same, that is, in trials where they saw the experimenter's hand performing the same movement as required by the instruction: in this condition, they misjudged the hand as theirs in about $30 \%$ of cases.

It is also in this particular condition that the rate of incorrect responses increased in schizophrenic patients. The error rate amounted to $77 \%$ in the group of patients with hallucinations or $80 \%$ in the group with delusional experiences, whereas in the non-hallucinating group, it was around $50 \%$. The fact that all patients gave nearly correct responses in the other two conditions (the error rate remained within 1-7\%) shows that the effect observed in trials Experimenter Same was not due to factors unrelated to the task, such as lack of attention, perceptual deficits or a perseverative bias when task demands increase. Seventeen patients accepted to undergo a further session of testing in which their basic perceptual abilities as well as their capacity to make same-different judgements was examined. In a subset of the BORB (Riddoch and Humphreys, 1993), the patients were asked to perform fine-grained analysis of the physical features of the stimulus in order to accomplish the task. All the patients performed well within the normal range in these tests. That is, their ability to process basic properties of visual stimuli, as well as to derive viewpoint-invariant information was unimpaired. Moreover, even when the task was of a remarkable complexity, no bias towards one type of response was found. Therefore the explanation for the peculiar distribution of the responses in the agency attribution experiment should be found in a deficit of the mechanism which is normally used for controlling and recognising one's own movements.

The discussion below examines several hypotheses for explaining the specific increase in error rate observed in the condition Experimenter Same. The first hypothesis relates to the corollary discharge model already alluded to in the introduction. According to this model (Sperry, 1950; von Holst, 1954), a comparison is made within the central nervous system, between an internally generated signal (which 
reflects the desired movement) and other central signals, anticipating the consequences of this movement. The study of visuomotor behaviour in normal subjects clearly supports the existence of such a mechanism for accounting for the generation of fast and automatic correcting movements during reaching at small targets, for example (see Jeannerod, 1988 for review).

The pattern of responses that we recorded in our condition Experimenter Same (in both normal controls and schizophrenics) could be explained by the paucity of movement related cues available to the comparator mechanism. As the subject's (invisible) hand and the experimenter's (visible) hand both executed the same movement, no mismatch occurred between the anticipated and the perceived final hand postures. The only available cues were the dynamic signals generated during the movements themselves. Slight differences in timing and kinematic pattern between the intended movement and that perceived by visual and kinaesthetic channels had to be used by the comparator in order to give the correct response. A decrease in sensitivity of this mechanism would explain the greater difficulties met by the schizophrenic patients. If this explanation were correct, however, the same patients should have difficulties in other visuomotor tasks involving a comparison between an intended movement and reafferent visual cues. Frith and Done (1989) reported absence of corrections in schizophrenics in tracking tasks, but only when visual feedback from the manipulandum was precluded (see also Malenka et al., 1982). This result shows that the patients were unable to match internal cues about movement direction with the anticipated outcome, but not that they were unable to use actual reafferent signals from the movement. In fact, a recent experiment performed by Fourneret et al. (unpublished data) shows that schizophrenic patients are not impaired in making accurate corrections in a difficult visuomotor task involving a conflict between the visual and the kinaesthetic reafferences from their movement (see also Pitblado et al., 1980).

The Frith model of schizophrenia (Frith, 1987, 1992) places the main emphasis on the disturbance of automatic processes as responsible for the failure of patients in making a conscious distinction between their own actions and those performed by others. In fact, there is evidence that automatic processes, such as implicit memory, priming etc., are usually left intact in schizophrenic patients (Callaway and Naghdi, 1982; Gras-Vincendon et al., 1994), whereas conscious cognitive processes (explicit memory, action planning) are known to be more severely affected (e.g. Gray et al., 1991). Accordingly, the present experiment was deliberately undertaken within a different conceptual framework, that of a dissociation between different behavioural modalities. There is abundant evidence in normal subjects that actions can be adequately performed without being consciously monitored. Subjects may correct visuomotor errors toward targets that they have not perceived (e.g. Bridgeman et al., 1981; Goodale et al., 1986). They may also make perceptual judgements on visual objects, that are in contradiction with their movements directed towards the same objects (e.g. Gentilucci and Negrotti, 1994; Gentilucci et al., 1996). More recently, Fourneret and Jeannerod (unpublished data) reported in normal subjects a similar dissociation between correct automatic tracking in a visuomotor task and 
erroneous conscious reports on what the subjects thought their hand had done to achieve these corrections.

These two levels of processing action-related events refer to widely different behavioural modalities (Jeannerod, 1997). Generating an action in the visuomotor context (reaching a predetermined visual goal, for example) implies that the representation of the action is transferred into execution mechanisms. Thus, the spatial (extrinsic) co-ordinates of the goal have to be transformed into a set of commands coded into a body-centred (intrinsic) frame of reference. This operation is the condition for the effector (e.g. the arm) to reach the goal. Such an operation is likely to result from a comparison, within the subject, between the internal model of the action and its sensory outcome. According to the above corollary discharge model, it is sufficient for the motor system to monitor the degree of match between the motor commands (representing the internal model of the goal) and peripheral signals arising from the movement, and to set a threshold beyond which a correction has to be produced. Note that this mechanism has to remain 'private' to be effective, for the reason that the reafferent signals can only be adequately used by the comparison mechanism if they refer to a movement which has been produced by the same system.

Generating a judgement on an action, on the other hand, is distinct from execution mechanisms. It relies on a comparison between the internal model of the goal and the perceptual (mostly visual) configuration of the environment. This comparison therefore does not necessitate transforming the co-ordinates of the goal into an intrinsic, motor code. Indeed, the visual configuration may arise from several sources and can result from an action performed by the observer, but also by other people. The problem is therefore, not only to determine whether the intended action was correctly achieved or not (which is the role of the above automatic mechanism), it is to determine where the configuration resulting from the action originated from. This perceptual judgement is likely to be made on the basis of central representations coded in a set of co-ordinates used for perception, that is, coordinates that relate to the external environment. At variance with what happens with the above 'private' mechanism, this set of co-ordinates will be shared by other agents and/or observers attending the same visual scene (see Frith, 1995).

This notion of shared representations therefore raises the key problem of the present experiment: how are representations pertaining to actions from different origins (and from different agents) distinguished from one another? And how are actions attributed to their veridical source? These critical issues have been raised in recent PET experiments in normal subjects. Conditions where subjects were intending actions and preparing for execution, mentally simulating actions, or observing actions performed by other people were compared (Decety et al., 1994, 1997; Grafton et al., 1996; Rizzolatti et al., 1996a; Stephan et al., 1995). The outcome of these studies is two-fold: First, there exists a cortical network common to all conditions, to which the inferior parietal lobule (area 40), the ventral premotor area (ventral area 6), and part of SMA contribute; second, motor representations for each individual condition are clearly specified by the activation of cortical zones which do not overlap between conditions. Area 4 is one of those: it is activated during 
motor execution and motor imagination, not during observation. Conversely, prefrontal areas in the middle and inferior frontal gyri (areas 45 and 46) are strongly activated during simulation and observation, but not during execution. In addition, even within overlapping zones, there are indications that both the extent and the precise topography of the activated areas may differ between conditions: This seems to be the case at least for SMA, where the activation during imagination is more rostral than during execution (Picard and Strick, 1996).

One of these cortical zones, located in the posterior and inferior part of the frontal lobe at the junction between the precentral and the inferior frontal gyri (the frontal operculum) deserves special attention. According to Rizzolatti et al. (1996b), this area, at the border between the ventral part of area 6 and areas 44 and 45, is homologous to a monkey area (area F5) involved in a particular form of movement representation. Neurones herein are activated whether the monkey executes a given hand movement or watches the same movement performed by another monkey. This property illustrates the close relationships of the two modalities of motor representation for intending and recognising actions, respectively. In humans, the ability of making agency judgements could rely on a similar mechanism, provided additional activity from other cortical areas would signal the intentional state of the agent. This activity would be the cue for referring the perceptual configuration resulting from the action to an internal origin, when it is present, or to an external source, when it is not.

It remains to be determined how the difficulties met by schizophrenic patients in recognising movements of uncertain origin is compatible with this framework. In our condition Experimenter Same, normal subjects misjudged the ownership of the experimenter's hand in about $30 \%$ of the trials. This finding suggests that the mechanism for recognising actions and attributing them to their true origin operates with a relatively narrow safety margin: in conditions where the visual cues are degraded or ambiguous, it is barely sufficient for making correct judgements about the origin of action, although it remains compatible with correct determination of agency in everyday life.

Deterioration of this mechanism leads to a situation where agency is systematically misjudged, as was the case in our patients with hallucinations and delusion of control. The consequence of this situation is attributing to oneself actions that pertain to someone else. Different degrees of deterioration would account for different degrees of severity of the symptoms created by mis-attributions of action. In the present case, patients failed to detect presentation of the alien hand only in the condition Experimenter Same. Virtually no errors were recorded when the patients saw their own hand. Clinicians report that most hallucinating patients show a tendency to incorporate external events in their own experience, or to interpret environmental cues as specifically directed to themselves. The peculiar error pattern that emerged from the present experiment is consistent with such a behaviour.

At present there is no direct evidence as to the nature of the mechanism, the alteration of which would create these problems in schizophrenic patients. If the above hypothesis that attribution of an action to the self or to another person is made on the basis of specific cortical activation patterns, then a form of comparison by 
'subtraction' between activation patterns in different conditions could be used to detect neural signals originating from non-overlapping areas. In schizophrenia, by contrast, the cortical activation related to cognitive motor states would become less selective, with the consequence that intending, observing or imagining an action would lead to indistinguishable patterns.

\section{Acknowledgements}

We thank Professor M. Marie-Cardine and Drs. T. d'Amato, J. Furtos, G. Burloux, J. Dubuis, A. Parriaud and B. Stalloni for referring the patients to us. Mr. P. Monjaud and M. Dittmar were involved in building the apparatus. J.L. Borach provided photographic assistance. We also gratefully acknowledge the thoughtful comments of G. Rizzolatti (Parma) and P. Livet (Aix-en-Provence) on an earlier version of this paper. Work supported by a grant from GIS Sciences de la Cognition, Paris. E.D. was supported by a fellowship from ESF, Strasbourg. N.G. is associated with I.T.T.A.C., 9 rue des Teinturiers, BP2116, Villeurbanne, 69616 Cedex.

\section{References}

Andreasen, N.C., 1983. The Scale for Assessment of Negative Symptoms (SANS). The University of Iowa, Iowa City, IA.

Andreasen, N.C., 1984. The Scale for Assessment of Positive Symptoms (SAPS) The University of Iowa, Iowa City, IA.

Bridgeman, B., Kirch, M., Sperling, A., 1981. Segregation of cognitive and motor aspect of visual function using induced motion. Perception and Psychophysics 29, 336-342.

Callaway, E., Naghdi, S., 1982. An information processing model for schizophrenia. Archives of General Psychiatry 39, 339-347.

Creutzfeldt, O., Ojeman, G., Lettich, E., 1989. Neuronal activity in the human lateral temporal lobe: II. Responses to own voice. Experimental Brain Research 77, 475-489.

Decety, J., Perani, D., Jeannerod, M., et al., 1994. Mapping motor representations with PET. Nature 371, 600-602.

Decety, J., Grezes, J., Costes, et al., 1997. Brain activity during observation of action. Influence of action content and subject's strategy. Brain 120, 1763-1777.

De Renzi, E., Vignolo, L.A., 1962. The Token Test: a sensitive test to detect receptive disturbances in aphasia. Brain 85, 665-678.

Frith, C.D., 1987. The positive and negative symptoms of schizophrenia reflect impairments in the perception and initiation of action. Psychological Medicine 17, 631-648.

Frith, C.D., 1992. The Cognitive Neuropsychology of Schizophrenia. Lawrence Erlbaum Associates, Hove, UK.

Frith, C.D., 1995. Consciousness is for other people. Behavioural and Brain Sciences 18, 682-683.

Frith, C., 1996. The role of the prefrontal cortex in self-consciousness: the case of auditory hallucinations. Philosophical Transactions of the Royal Society London B 351, 1505-1512.

Frith, C.D., Done, D.J., 1989. Experiences of alien control in schizophrenia reflect a disorder in the central monitoring of action. Psychological Medicine 19, 359-363.

Gentilucci, M., Negrotti, A., 1994. Dissociation between perception and visuomotor transformation during reproduction of remembered distances. Journal of Neurophysiology 72, 2026-2030.

Gentilucci, M., Chieffi, S., Daprati, E., Saetti, M.C., Toni, I., 1996. Visual illusion and action. Neuropsychologia 34, 369-376. 
Goodale, M.A., Pélisson, D., Prablanc, C., 1986. Large adjustments in visually guided reaching do not depend on vision of the hand or perception of target displacement. Nature 320, 748-750.

Grafton, S.T., Arbib, M.A., Fadiga, L., Rizzolatti, G., 1996. Localisation of grasp representations in humans by positron emission tomography. 2. Observation compared with imagination. Experimental Brain Research 112, 103-111.

Gras-Vincendon, A., Danion, J.M., Grangé, D., et al., 1994. Explicit memory, repetition priming and cognitive skill learning in schizophrenia. Schizophrenia Research 13 (2), 117-126.

Gray, J.A., Feldon, J.N., Rawlins, J.N.P., Hemsley, D.R., Smith, A.D., 1991. The neuropsychology of schizophrenia. Behavioural and Brain Sciences 14, 1-84.

von Holst, E., 1954. Relations between the central nervous system and the peripheral organs. British Journal of Animal Behaviour 2, 89-94.

Jeannerod, M., 1988. The neural and behavioural organisation of goal-directed movements. Oxford University Press, Oxford, UK:

Jeannerod, M., 1997. The cognitive neuroscience of action. Blackwell, Oxford, UK.

Lecrubier, Y., Sheehan, D., Weiller, E., et al., 1997. The MINI International neuropsychiatric interview (MINI). A short diagnostic structured interview: reliability and validity according to the CIDI. European Psychiatry 12 (5), 217-224.

Leslie, A.M., 1987. Pretence and representation: the origins of 'theory of mind'. Psychological Review 94, 412-426.

Malenka, R.C., Angel, R.W., Hampton, B., Berger, P.A., 1982. Impaired central error-correcting behaviour in schizophrenia. Archives of General Psychiatry 39, 101-107.

McGuire, P.K., Silbersweig, D.A., Wright, I., Murray, R.M., Frackowiak, R.S.J., Frith, C.D., 1996. The neural correlates of inner speech and auditory verbal imagery in schizophrenia: relationship to auditory verbal hallucinations. British Journal of Psychiatry 169, 148-159.

Meltzoff, A.N., 1995. Understanding the intentions of others. Re-enactment of intended acts by 18-monthold children. Developmental Psychology 31, 838-850.

Nielsen, T.I., 1963. Volition: A new experimental approach. Scandinavian Journal of Psychology 4, $225-$ 230.

Oldfield, R.C., 1971. The assessment and analysis of handedness: The Edinburgh Inventory. Neuropsychologia 9, 97-113.

Picard, N., Strick, P.L., 1996. Motor areas of the medial wall: a review of their location and functional activation. Cerebral Cortex 6, 342-353.

Pitblado, C., Shapiro, J., Petrides, M., 1980. Adaptation to prismatic displacement by schizophrenics and normals. Neuropsychobiology 6, 201-207.

Riddoch, M.J., Humphreys, G.W., 1993. BORB: Birmingham Object Recognition Battery. Lawrence Erlbaum Associates, Hove, UK.

Rizzolatti, G., Fadiga, L., Matelli, et al., 1996a. Localisation of grasp representations in humans by PET. 1. Observation versus execution. Experimental Brain Research 111, 246-252.

Rizzolatti, G., Fadiga, L., Gallese, V., Fogassi, L., 1996b. Premotor cortex and the recognition of motor actions. Cognitive Brain Research, 3, 131-141.

Silbersweig, D.A., Stern, E., Frith, C., 1995. A functional neuroanatomy of hallucinations in schizophrenia. Nature 378, 176-179.

Sperry, R.W., 1950. Neural basis of the spontaneous optokinetic response produced by visual inversion. Journal of Comparative and Physiological Psychology 43, 482-489.

Stephan, K.M., Fink, G.R., Passingham, R.E., et al., 1995. Functional anatomy of the mental representation of upper extremity movements in healthy subjects. Journal of Neurophysiology 73, 373-386.

Wechsler, D., 1981. Échelle d'Intelligence pour Adultes (WAIS). Paris: Editions du Centre de Psychologie Appliquée, Paris.

Wolpert, D.M., Ghahramani, Z., Jordan, M., 1995. An internal model for sensorimotor integration. Science $269,1880-1882$. 